EPJ Web of Conferences 11, 05006 (2011)

DOI:10.1051/epjconf/20111105006

(C) Owned by the authors, published by EDP Sciences, 2011

\title{
The transiting exoplanet CoRoT-11b and its peculiar tidal evolution
}

\author{
D. Gandolfi ${ }^{1,2}$, A.F. Lanza ${ }^{3}$, C. Damiani ${ }^{3}$, and the CoRoT Exoplanet \\ Science Team
}

${ }^{1}$ Research and Scientific Support Department, European Space Agency, PO Box 299, Keplerlaan 1, 2200 AG Noordwijk, The Netherlands

[davide.gandolfi@esa.int]

2 Thüringer Landessternwarte, Sternwarte 5, Tautenburg, D-07778 Tautenburg, Germany

${ }^{3}$ INAF-Osservatorio Astrofisico di Catania, Via Santa Sofia 78, I-95123

Catania, Italy

\begin{abstract}
CoRoT-11b is a fairly massive hot-Jupiter $\left(M_{\mathrm{p}}=2.33 \pm 0.34 \mathrm{M}_{\mathrm{Jup}}\right)$ in a 3 days orbit around a F6 V star with an age of $2 \pm 1$ Gyr. The relatively high projected rotational velocity of the $\operatorname{star}\left(v \sin i_{\star}=40 \pm 5 \mathrm{~km} / \mathrm{s}\right)$ places CoRoT-11 among the most rapidly rotating planet hosting stars discovered so far. Assuming that the star is seen equator-on, the $v \sin i_{\star}$ and the star radius $\left(\mathrm{R}_{*}=1.37 \pm 0.03 R_{\odot}\right)$ translate into a stellar rotation period of $1.73 \pm 0.26$ days. This peculiar planet/star configuration offers an unique opportunity to study the tidal evolution of the system. Owing to the strong tidal interaction, the planet would have moved outwards, from a starting semi-major axis corresponding to an orbital period almost synchronized with the stellar rotation. We found that the present value of the tidal quality factor $Q_{\mathrm{s}}^{\prime}$ could be measured by a timing of the mid-epoch of the transits to be observed with an accuracy of about $0.5-1$ seconds over a time baseline of about 25 years.
\end{abstract}

\section{Introduction}

Transiting extrasolar planets are cornerstones for understanding the nature of planets beyond the solar system, since a wealth of precious information can be gained (Winn 2010). The geometry of transiting planets allows us to study them in significantly more detail than the remaining majority of the planet sample. Indeed, the planetary radius and mass can be derived by combining transit photometric observations with radial velocity (RV) measurements of the host star. Transits offer a unique opportunity to measure the sky-projected angle $(\lambda)$ between the orbital angular momentum vector and the spin axis of the star (Gaudi \& Winn 2007), by detecting the Rossiter-McLaughlin $(\mathrm{RM})$ effect, i.e., the spectral distortion observed in the line profile of a rotating star as a second object passes in front of the stellar disc.

Systems with transiting planets can also be used to test tidal interaction theories and better understand the tidal dissipation efficiency, which is usually parameterized by a dimensionless quality factor $Q^{\prime}$ (e.g., Zahn 2008). So far, the efficiency of tidal

This is an Open Access article distributed under the terms of the Creative Commons Attribution-Noncommercial License 3.0, which permits unrestricted use, distribution, and reproduction in any noncommercial medium, provided the original work is properly cited. 
Table 1.: CoRoT-11b - Star and planet physical parameters

\begin{tabular}{cccccc}
\hline $\begin{array}{c}M_{*} \\
\left(\mathrm{M}_{\odot}\right)\end{array}$ & $\begin{array}{c}R_{*} \\
\left(\mathrm{R}_{\odot}\right)\end{array}$ & $\begin{array}{c}v \sin i_{\star} \\
(\mathrm{km} / \mathrm{s})\end{array}$ & $\begin{array}{c}M_{\mathrm{p}} \\
\left(\mathrm{M}_{\mathrm{J}}\right)\end{array}$ & $\begin{array}{c}R_{\mathrm{p}} \\
\left(\mathrm{R}_{\mathrm{J}}\right)\end{array}$ & $\begin{array}{c}P_{\text {orb }} \\
(\text { days })\end{array}$ \\
\hline $1.27 \pm 0.05$ & $1.37 \pm 0.03$ & $40 \pm 5$ & $2.33 \pm 0.34$ & $1.43 \pm 0.03$ & 2.9943 \\
\hline \hline
\end{tabular}

dissipation has been constrained for close binary systems in stellar clusters of different ages, by measuring the orbital period below which the orbits appear to have been circularized. The observations reviewed by Ogilvie \& Lin (2007) indicate that an average $Q^{\prime}$ ranging between $5 \times 10^{5}$ and $2 \times 10^{6}$ is adequate to account for the circularization of late-type main-sequence binaries. Systems with transiting planets have the best determined stellar and planetary parameters and are thus particularly suited to study tidal dissipation. Among them, F-type host stars having a mass $M \geq 1.2-1.5 \mathrm{M}_{\odot}$ evolve quite fast during their main-sequence lifetime, thus improving significantly their age estimate from model isochrone fitting in comparison with lower mass stars. A good age estimate is important to constrain the average value of $Q^{\prime}$ by modeling the tidal evolution of a particular system.

An ideal extrasolar planet system to better constrain the quality factor $Q^{\prime}$ is CoRoT-11, a fairly massive, transiting Jupiter-like planet in a relatively short-period orbit around a rapidly rotating F6 dwarf star (Gandolfi et al., 2010). The peculiar ratio of the rotation period of the star to the orbital period of the planet is presently the smallest among systems having a radial velocity curve compatible with zero eccentricity, and this makes CoRoT-11 a suitable system to investigate the tidal interaction between the planet and its host star. The tidal interaction time-scale of CoRoT-11 is fully compatible with the main-sequence lifetime of the system, a unique case among the transiting extra solar planets.

In the present paper we report on a preliminary study of the tidal evolution of the CoRoT-11 system and demonstrate how the present value of the tidal quality factor of the star $\left(Q_{\mathrm{s}}^{\prime}\right)$ can be directly measured with suitable transit observations extended on a time interval of a few decades. A more thorough study will be the subject of a forthcoming paper (Lanza et al., 2011, in preparation).

\section{The CoRoT-11 system parameters}

In a recent paper, Gandolfi et al. (2010) presented the observations leading to the discovery of CoRoT-11 and derived the parameters of the system (Table 1). The stellar rotation period can be inferred only from the projected rotational velocity $\left(v \sin i_{\star}=40 \pm 5 \mathrm{~km} / \mathrm{s}\right)$ and the estimated radius of the $\operatorname{star}\left(\mathrm{R}_{*}=1.37 \pm 0.03 R_{\odot}\right)$ as no evidence of rotational modulation compatible with the measured $v \sin i_{\star}$ was found in the CoRoT light curve. A modulation with a period of about 8.5 days was detected in the CoRoT data but it is incompatible with the expected maximum rotation period of $1.73 \pm 0.25$ days as derived from $v \sin i_{\star}$ and $R_{*}$. It is likely to arise from a nearby contaminant star about $2^{\prime \prime}$ from CoRoT-11 that falls inside the CoRoT photometric mask.

The eccentricity of the planetary orbit is poorly constrained by the RV curve alone due to the uncertainty of the RV measurements for such a rapidly rotating F-type star. Nevertheless, an upper limit of 0.2 was derived from the mean stellar density as inferred 


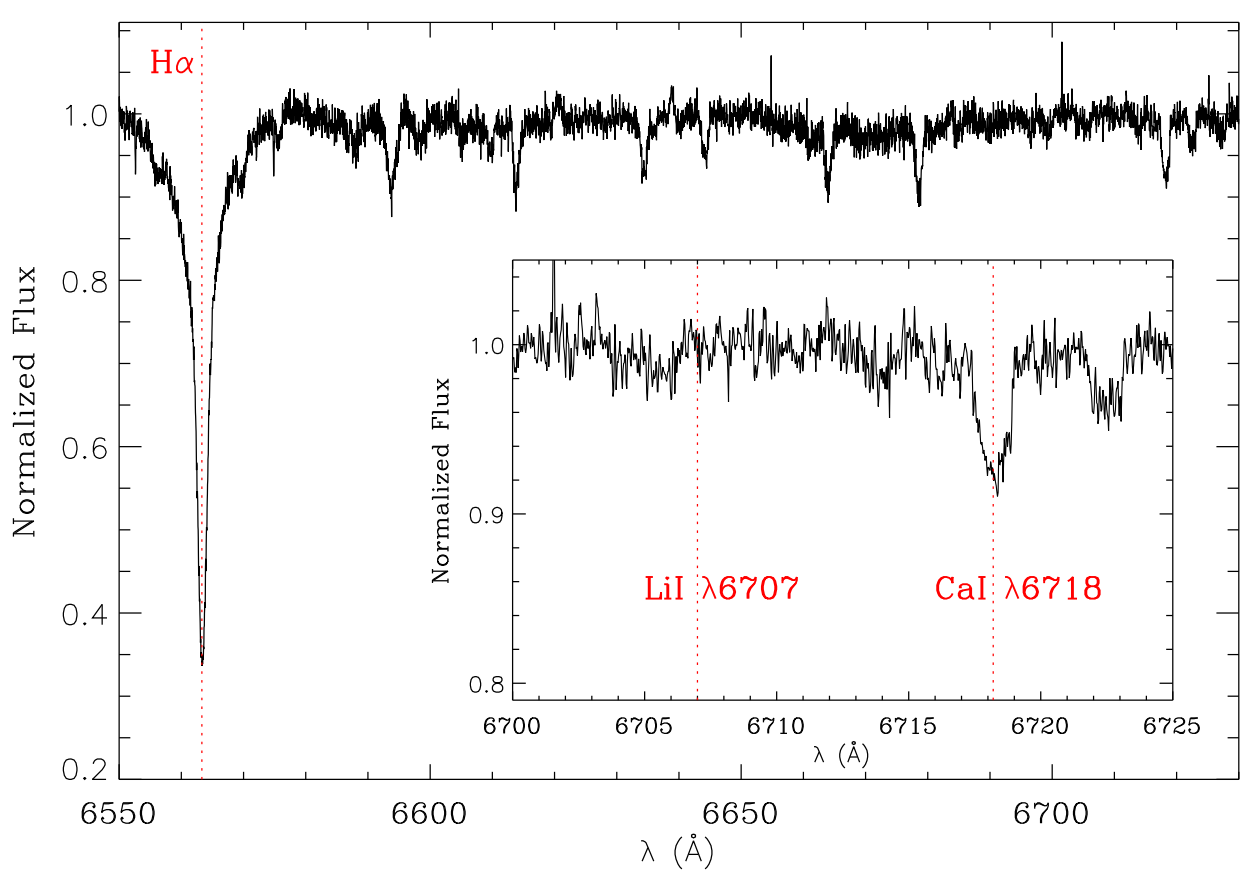

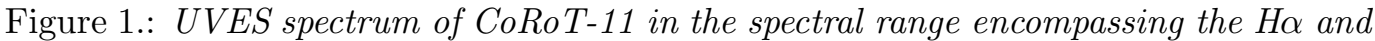
$L i \mathrm{I} \lambda 6708 \AA$ lines. Note the absence of the LiI $\lambda 6708 \AA$ line in the inset of the figure.

from the modeling of the transit light curve. Indeed, an eccentricity value larger than 0.2 would lead to a mean stellar density incompatible with a F6 V star. In any case, the RV measurements and transit data presented in Gandolfi et al. (2010) are fully compatible with a circular orbit.

The observations of part of the RM effect allowed the authors to assess that the orbit of CoRoT-11b is prograde, but unfortunately were not sufficient to establish the value of the angle $\lambda$. An aligned system, i.e., having $\lambda=0^{\circ}$, is compatible with their observations.

Concerning the age of the system two solutions were found: (1) a solution in which the planet hosting star is slightly evolved from the main sequence, with an age of about $2.0 \pm 1.0 \mathrm{Gyr}$ (ii) a very young solution in which the star is still on the pre-main sequence (PMS) phase, with an age of about $12 \pm 2 \mathrm{Myr}$. However, the latter scenario is at variance with the lack of a detectable Li I $\lambda 6708 \AA$ line in the spectrum of CoRoT-11 (Fig. 1). According to the effective temperature of the star $\left(T_{\text {eff }}=6440 \pm 120 \mathrm{~K}\right)$, CoRoT-11 would belong to the narrow class of F-stars which have suffered strong surface lithium depletion during the first billion years of their life. Indeed, studies of the lithium content in the photosphere of F-type stars in galactic clusters and field stars have revealed the presence of a narrow dip in the lithium abundance for effective temperature between 6500 and $6800 \mathrm{~K}$ (Mallik, Parthasarathy, \& Pati 2003; BöhmVitense 2004). An age of $2.0 \pm 1.0 \mathrm{Gyr}$ is thus more plausible for the planet-star system. 


\section{EPJ Web of Conferences}

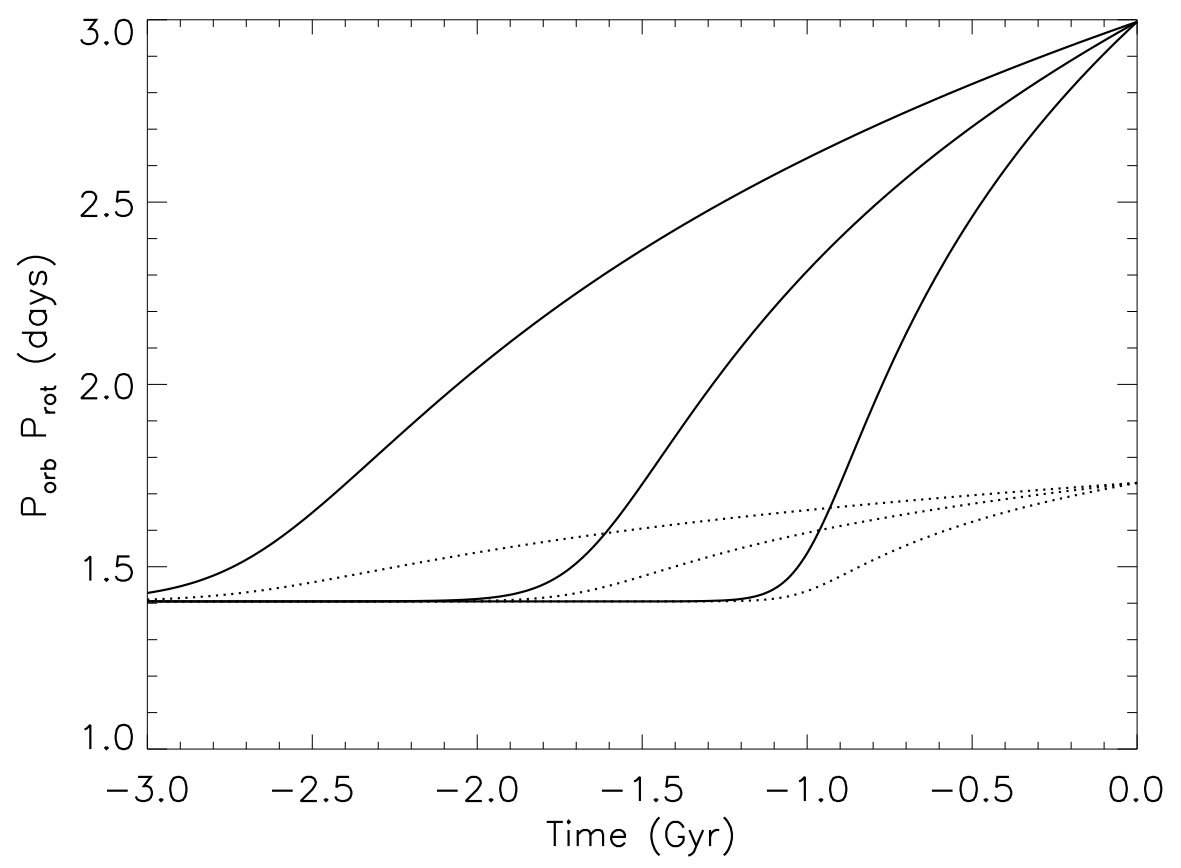

Figure 2.: The backward evolution of the orbital period $P_{\text {orb }}$ (solid line) and the rotation period $P_{\text {rot }}$ (dotted line) vs. time since the present epoch for different values of the modified tidal quality factor $Q_{\mathrm{s}}^{\prime}$. From left to right, the values of $Q_{\mathrm{s}}^{\prime}$ are: $8 \times 10^{6}$, $5 \times 10^{6}$, and $3 \times 10^{6}$, respectively.

\section{Backward tidal evolution of the system}

We adopted the classic equilibrium tidal model after Hut (1981), in the formulation recently given by Leconte et al. (2010), and integrated the tidal evolution equations backwards in time starting from the present condition, i.e. stellar rotation period $P_{\text {rot }}=1.73$ days, planet orbital period $P_{\text {orb }}=2.9943$ days, star mass $M=1.27 \mathrm{M}_{\odot}$, star radius $R=1.37 \mathrm{R}_{\odot}$, planet mass $M_{\mathrm{p}}=2.33 \mathrm{M}_{\mathrm{J}}$, planet radius $R_{\mathrm{p}}=1.43 \mathrm{R}_{\mathrm{J}}$, orbital eccentricity $e=0$, stellar obliquity $\epsilon=0^{\circ}$, and adopting a stellar quality factor $Q_{\mathrm{s}}^{\prime}$ ranging from $3 \times 10^{6}$ to $8 \times 10^{6}$. Note that the results do not depend on the planet quality factor $Q_{\mathrm{p}}^{\prime}$, provided that the orbital eccentricity and stellar obliquity are zero, and the planet is assumed to be in the pseudo-synchronous rotation state.

As shown in Fig. 2, the backward evolutions of the orbital period of the planet and rotation period of the star approach asymptotically a synchronous state corresponding to $P_{\text {rot }}=P_{\text {orb }} \simeq 1.4$ days, regardless of the adopted stellar quality factor $Q_{\mathrm{s}}^{\prime}$. The angular momentum is exchanged between the stellar spin and the planet, but the rotation period of the star changes only slightly because its moment of inertia is significantly greater than that of the planetary orbit. 
Since the estimated age of CoRoT-11 ranges from 1 to $3 \mathrm{Gyr}$, one is tempted to constrain the value of the stellar modified quality factor as $3 \times 10^{6} \leq Q_{\mathrm{s}}^{\prime} \leq 8 \times 10^{6}$, which is about one order of magnitude smaller than the average value predicted by Barker \& Ogilvie (2009). However, this conclusion is valid only if the synchronous state found by our backward integration is actually the initial state of the system when it settled on the ZAMS and if its evolution proceeded with $e=0$ and $\epsilon=0^{\circ}$. Therefore, we need to investigate in more detail the possible initial state of the system to prove whether such a synchronous state is indeed a possible initial condition.

\section{The initial state}

According to the currently considered planet formation scenario, hot Jupiters are formed at several AUs from their stars, beyond the snow line where volatile elements can condense, and then migrate toward their stars on a timescale not exceeding $10^{5}-10^{6}$ yr (Papaloizou \& Terquem 2006). As recently proposed by Lanza (2010), the stellar magnetic field might in some case halt the inward migration of the planet at the co-rotation radius and synchronize stellar and planetary orbital rotation. If the magnetic field of the PMS star is strong enough $(\approx 3-5 \mathrm{kG})$ it might effectively couple the orbital motion of the planet to the rotation of the star even after the disc has disappeared (Lovelace, Romanova, \& Barnard 2008; Vidotto et al 2010). In this case, a quasi-synchronous state is obtained on a timescale of $\approx 2-3 \mathrm{Myr}$ and maintained until the field strength decreases rapidly as the star gets close to the ZAMS. Eventually, the star settles on the ZAMS and its final contraction leads to $P_{\text {rot }} \lesssim P_{\text {orb }}$, so tides will push the planet away from the star.

The scenario described above might have been the initial condition for CoRoT-11 and this should justify the initial synchronous state found in Sect. 3 by the backwards integration of the tidal equations. Furthermore, according to Lanza (2010), CoRoT11 would have suffered a negligible angular momentum loss during its main-sequence lifetime thanks to the spectral type of the star and the presence of its hot-Jupiter. Indeed, the star-planet interaction should have led to a compact corona with mainly closed field lines, producing a very small loss of angular momentum through the stellar wind.

\section{Discussion}

An interesting opportunity offered by CoRoT-11 is the possibility to measure the present value of $Q_{\mathrm{s}}^{\prime}$ from the rate of orbital period variation. If the present orbit is circular, the semi-major axis is subject to a secular increase because the star is rotating faster than the orbital motion of the planet, so angular momentum is transferred from the stellar spin to the orbit via the tidal interaction. When $e=0$ and $\epsilon=0^{\circ}$, the rate of such a transfer depends on the system parameters, that are known to a good level of precision, and on $Q_{\mathrm{s}}^{\prime}$ only. Therefore, we can compute the rate of orbital period increase $d P_{\text {orb }} / d t$ and use it to derive the difference $(O-C)$ between the observed time of mid-transit and that computed with a constant period ephemeris after $N$ orbital periods. Using the tidal evolution model of Leconte et al. (2010) and assuming $e=0$, $\epsilon=0^{\circ}$ and a stellar rotation period $P_{\text {rot }}=1.73$ days, we found:

$$
(O-C)=8.34\left(\frac{Q_{\mathrm{s}}^{\prime}}{10^{6}}\right)^{-1} \mathrm{~s}
$$


after $N=3000$ orbital period, i.e., 24.6 years. Taking into account the magnitude of CoRoT-11 $(V=12.9 \mathrm{mag})$, the depth of the transit $(0.011 \mathrm{mag})$, and the very-low level of magnetic activity of the star (Gandolfi et al., 2010), it is reasonable to assume that a timing accuracy of $\sim 5$ seconds can be easily reached from the ground with a 10 m-class telescope. This timing accuracy would improve up to $\sim 0.5-1$ seconds with a space-borne dedicated telescope of aperture 2-3 $\mathrm{m}$, since the precision is expected to scale with the accuracy of the photometry. Such an instrument would allow us to detect the expected transit time variation (TTV) with $3 \sigma$ significance after an interval of $\sim 25$ years for $Q_{\mathrm{S}}^{\prime} \sim 5 \times 10^{6}$. Even in the case that no significant TTV is detected, a stringent lower limit can be placed on $Q_{\mathrm{s}}^{\prime}$, with the sensitivity increasing as the square of the considered time baseline.

\section{References}

Barker, A. J., \& Ogilvie, G. I. 2009, MNRAS, 395, 2268

Böhm-Vitense, E. 2004, AJ, 128, 2435

Gandolfi, D., Hébrard, G., Alonso, R., et al. 2010, A\&A, in press, DOI: 10.1051/00046361/201015132 arXiv: 1009.2597

Gaudi, B. S., \& Winn J. N 2007, ApJ, 655, 550

Hut, P. 1981, A\&A, 99, 126

Lanza, A. F. 2010, A\&A, 512, A77

Leconte, J., Chabrier, G., Baraffe, I., \& Levrard, B. 2010, A\&A, 516, A64

Lovelace, R. V. E., Romanova, M. M., \& Barnard, A. W. 2008, MNRAS, 389, 1233

Ogilvie, G. I., \& Lin, D. N. C. 2007, ApJ, 661, 1180

Mallik, S. V., Parthasarathy, M., \& Pati, A. K. 2003,A\&A, 409, 251M

Papaloizou, J. C. B., Terquem, C. 2006,Reports on Progress in Physics, 69, 119

Vidotto, A. A., Opher, M., Jatenco-Pereira, V., \& Gombosi, T. I. 2010, ApJ, 720, 1262

Winn, J. N. 2010, eprint arXiv:1001.2010

Zahn, J.-P. 2008, EAS Publications Series, 29, 67 